\title{
DIFICULDADES ENFRENTADAS PELOS ENFERMEIROS NO MANEJO DOS PACIENTES COM HANSENÍASE
}

\section{DIFFICULTIES FACED BY NURSES IN THE MANAGEMENT OF LEPROSY PATIENTS}

\section{Ana Alinne Gomes da Penha ${ }^{1} *$ Jéssica Lima Soares $^{2} *$ Fernanda Maria Silva $^{3}$ Déborah Albuquerque Alves Moreira ${ }^{4} *$ Regina Petrola Bastos Rocha ${ }^{5} *$ Huana Carolina Cândido Moraes ${ }^{6}$}

\section{RESUMO}

Objetivo: conhecer as dificuldades enfrentadas pelos enfermeiros no manejo dos pacientes com hanseníase. Método: estudo exploratório, de caráter descritivo e qualitativo. A amostra é composta por nove enfermeiros que atuam na Estratégia Saúde da Família, em região endêmica do sul do Ceará, Brasil. A coleta de dados foi realizada com um roteiro de entrevista semiestruturada, nos meses de agosto a setembro de 2015, com posterior análise de conteúdo temático. Resultados: embasando-se nos resultados obtidos emergiram três categorias: dificuldades elencadas na realização do exame dermatoneurológico pelos enfermeiros da estratégia saúde da família; intercorrências no atendimento ao paciente com hanseníase; detecção precoce versus educação permanente. Considerações finais: o estudo revelou as dificuldades enfrentadas como a falta de instrumentos para realizar os testes dermatoneurológicos; estigma e preconceito manifestos pela população; detecção precoce com falhas de domínio; educação permanente pouco estruturada para atender a demanda dos profissionais. Ressalta-se a importância de mais investimento em pesquisas de inovação no manejo da hanseníase.

Palavras-Chave: Hanseníase; Enfermagem; Relações Enfermeiro-Paciente; Estratégia Saúde da Família; Atenção Primária à Saúde.

\begin{abstract}
Objective: know the difficulties faced by nurses in the management of patients with leprosy. Method: exploratory, descriptive and qualitative study. The sample is composed of nine nurses who work in the Family Health Strategy, in an endemic region of southern Ceará, Brazil. Data collection was performed using a semi-structured interview script, from august to september 2015, with subsequent analysis of thematic content. Results: based on the results obtained, three categories emerged: difficulties listed in the performance of the dermato-neurological examination by nurses of the family health strategy; complications in the care of patients with leprosy; early detection versus continuing education. Final Considerations: the study revealed the difficulties faced, such as the lack of instruments to perform dermato-neurological tests; stigma and prejudice manifests by the population; early detection with domain failures; permanent education poorly structured to meet the demands of professionals. The importance of more investment in innovation research in the management of leprosy is highlighted.

Keywords: Leprosy; Nursing; Nurse-Patient Relations; Family Health Strategy; Primary Health Care.

\footnotetext{
1 Enfermeira. Especialista em Saúde da Família. Universidade Regional do Cariri (URCA). Crato, Ceará, Brasil. E-mail: anaalinne.nurse@gmail.com ORCID: https://orcid.org/0000-0001-9253--1199

2 Enfermeira. Mestranda em Enfermagem. Universidade Regional do Cariri (URCA). Crato, Ceará, Brasil. E-mail: jessicalimasoares92@gmail.com.br ORCID: https://orcid.org/0000-0003-4247-8822

3 Enfermeira. Mestre em Saúde da Família. Universidade Regional do Cariri (URCA). Crato, Ceará, Brasil. E-mail: fernandamsmv@gmail.com ORCID: https://orcid.org/0000-0002-0504-9896

4 Enfermeira. Mestre em Enfermagem. Universidade Regional do Cariri (URCA). Crato, Ceará, Brasil. E-mail: dbrhalbuquerque@gmail.com ORCID: https://orcid.org/0000-0002-2823-8681

5 Enfermeira. Doutoranda em Ciências da Saúde. Faculdade de Medicina do ABC (FMABC), São Paulo, Brasil. E-mail: rpetrola@yahoo.com.br ORCID: https://orcid.org/0000-0003-0626-232X

6 Enfermeira. Doutora em Enfermagem. Universidade Federal do Ceará (UFC). Fortaleza, Ceará, Brasil. E-mail: huanacarolina@yahoo.com.br ORCID: http://orcid.org/0000-0001-6435-1457 


\section{INTRODUÇÃO}

A hanseníase, patologia infectocontagiosa, causada pelo Micobacterium leprae, é considerada uma doença negligenciada. O Brasil integra a lista global dos 22 países com alta carga da doença, ocupando o $2^{\circ}$ lugar com mais casos notificados no mundo, ficando atrás apenas da Índia (OMS $)^{1}$. Quando analisada por regiões, a distribuição geográfica da doença no país apresenta padrões desiguais, com uma alta concentração de casos novos nas regiões Centro-Oeste, Norte e Nordeste quando comparadas às regiões Sul e Sudeste ${ }^{2}$. Nas Américas, foram detectados 20.957 novos casos de hanseníase, com taxa de detecção de 3,08/100.000 habitantes, sendo 28.660 casos novos notificados no Brasil, representando 93\% dos registros de casos da doença em 2018. O Ceará identificou 1.691 casos novos de hanseníase em 2018, com taxa de detecção de 18,63 casos novos por 100.000 habitantes, considerada alta pelos parâmetros da $\mathrm{OMS}^{3}$.

No período de 2008 a 2019 foram notificados 23.622 casos novos da doença no estado do Ceará, sendo 1.292 em menores de 15 anos. Houve significativa redução na taxa de detecção geral de hanseníase $(43,6 \%)$, passando de 30,5/100.000 habitantes em 2008 para 17,2/100.000 habitantes em 2019 Esses dados apontam a necessidade de continuar com uma atuação efetiva e programada dos profissionais de saúde, especialmente enfermeiros da atenção primária à saúde de áreas endêmicas. Entretanto, são relatadas dificuldades pelos profissionais que são protagonistas nas ações para um enfrentamento mais eficaz, possível por meio da detecção precoce e do controle da doença.

A Atenção Primária à Saúde no Brasil está ancorada na atuação da Estratégia Saúde da Família, que possui um papel fundamental no controle da hanseníase, desenvolvendo ações e atividades para seu alcance, além de ser imprescindível para a reorientação do modelo assistencial e para a consolidação das diretrizes do Sistema Único de Saúde ${ }^{5}$. Ressalta-se a importância da atuação dos profissionais que compõem essa Estratégia, principalmente, médicos e enfermeiros, que devem estar atentos para realizar ações que quebrem a cadeia de transmissão da doença, favorecendo a detecção precoce de sinais e sintomas e o fortalecimento da cobertura dos exames de contatos ${ }^{6}$.

Acerca dos sinais e sintomas, as consequências clínicas da hanseníase não atingem somente a pele, suas manifestações envolvem desde as manchas hipocrômicas, acastanhadas ou avermelhadas, com alterações de sensibilidade, até o comprometimento de nervos superficiais da pele e troncos nervosos periféricos (localizados na face, pescoço, terço médio do 
braço e abaixo do cotovelo e dos joelhos), olhos e órgãos internos (mucosas, testículos, ossos, baço, fígado etc.), podendo acarretar à pessoa acometida deficiências e incapacitações incontornáveis pelo resto da vida $^{7}$.

O controle da doença está centrado na detecção precoce das manifestações clínicas, no tratamento instituído de forma correta e na identificação de contatos suscetíveis. Um indicador sensível da transmissão da doença é o diagnóstico em crianças ${ }^{8}$. Todas essas ações podem ser executadas na atenção primária à saúde pelo enfermeiro. Portanto, o enfermeiro tem papel imprescindível no monitoramento da doença quando realiza assistência ao paciente com suspeita ou diagnóstico confirmado da doença, porém, a manutenção de situações de endemia da doença em algumas regiões suscita o questionamento acerca da efetividade da ação desses profissionais. Em virtude disso, pretende-se responder a seguinte pergunta: quais as dificuldades enfrentadas pelos enfermeiros no manejo dos pacientes com hanseníase?

Conhecer a realidade de quem lida com o desafio diário da doença pode melhor subsidiar a prática profissional e a tomada de decisão nas dificuldades para o enfrentamento dessa problemática. Diante do exposto, a pesquisa tem por objetivo conhecer as dificuldades enfrentadas pelos enfermeiros no manejo dos pacientes com hanseníase. Esse conteúdo contribuirá para o desenvolvimento da assistência de enfermagem com maiores informações para os profissionais que atuam ou vão atuar no atendimento aos pacientes acometidos pela hanseníase, que monitoram e trabalham no controle do avanço dessa enfermidade.

\section{MÉTODO}

O estudo, do tipo exploratório, com abordagem qualitativa de caráter descritivo, foi realizado com os enfermeiros da Atenção Primária à Saúde (APS) de um município de pequeno porte da região endêmica do Cariri, sul do Ceará, Brasil.

O cenário do estudo constituiu-se de nove equipes da Estratégia Saúde da Família (ESF), sendo duas da zona urbana e sete da zona rural que correspondem à frente local responsável pelas atividades do Programa de Controle da Hanseníase em nível de APS. Os participantes da pesquisa foram nove enfermeiros que atuam na ESF do referido município. Foram adotados os seguintes critérios de inclusão: ter realizado acompanhamento de pelo menos um paciente com hanseníase até alta por cura e ser enfermeiro da ESF há mais de um ano. Já os critérios de exclusão adotados foram: ausência de casos notificados de hanseníase no território de abrangência da equipe que o enfermeiro atua e ter vínculos temporários. A coleta de dados foi realizada durante os meses de agosto a setembro de 2015 . 
$\mathrm{O}$ instrumento adotado foi um roteiro de entrevista semiestruturada, sendo utilizado gravador digital e, posteriormente, feito transcrição e digitação das falas. Antecedendo a entrevista, foi realizada a leitura e assinatura da autorização individual do Termo de Consentimento Livre e Esclarecido (TCLE). É importante esclarecer que, para zelar pela identidade dos participantes, foram utilizados códigos como a letra E (Enfermeiro) e números de identificação $1,2 \ldots 9$.

Empregou-se a técnica de análise de conteúdo temática a qual é dividida em três etapas: pré-análise, que corresponde à escolha do material a ser utilizado; exploração do material, que consiste na operação de codificação; tratamento dos resultados obtidos e interpretação, que coloca em relevo as informações obtidas, propõe inferências e realiza interpretações previstas no seu quadro teórico ${ }^{9}$.

$\mathrm{Na}$ análise de conteúdo temático dos dados emergiram três categorias, a saber: dificuldades elencadas na realização do exame dermatoneurológico pelos enfermeiros da ESF; intercorrências no atendimento ao paciente com hanseníase; detecção precoce versus educação permanente. $\mathrm{O}$ projeto foi submetido à apreciação do Comitê de Ética em Pesquisa da Universidade Regional do Cariri, obtendo parecer favorável (protocolo $\left.\mathrm{n}^{\mathrm{o}} 705.008\right)$.

\section{RESULTADOS E DISCUSSÃO}

O estudo foi composto por nove enfermeiros da ESF. Em relação ao perfil dos entrevistados, a faixa etária situou-se entre 24 e 34 anos, configurando-se adultos jovens em atuação. Em relação ao sexo, foi possível observar que $90 \%$ eram compostos pelo sexo feminino. O tempo de trabalho foi superior a dois anos de experiência em ESF para todos os entrevistados. No tocante à titulação, $100 \%$ dos participantes possuíam pós-graduação latu senso, porém a área de especialização foi variada, sendo que apenas um entrevistado possuía formação especializada em saúde da família. A seguir, são apresentados os trechos das entrevistas que contemplam as três categorias e a discussão com a literatura pertinente.

\section{Dificuldades elencadas na realização do exame dermatoneurológico pelos enfermeiros da ESF}

A ESF é desenvolvida em unidades básicas que atuam na sua área de concentração que abrangem todo o território ao qual fazem parte. Tais unidades devem dispor de uma estrutura física e recursos adequados para o desenvolvimento das atividades inerentes aos serviços ofertados sem prejuízo ao atendimento, a fim de oferecer uma assistência acessível e de qualidade.

Ainda, essas unidades devem ser providas de local reservado e silencioso com 
materiais específicos que auxiliem os profissionais no desempenho de suas atividades de acordo com sua área. No tocante ao atendimento de pacientes com hanseníase, exemplos de tais materiais são: fio dental sem sabor com cera; tabela de Snellen; régua; lanterna clínica; canetas de várias cores; tubos de ensaios; chumaço de algodão; alfinete; monofilamentos/estesiômetros. Esses materiais são utilizados para realizar o exame dermatoneurológico que consiste em avaliar a sensibilidade da córnea, acuidade visual e as sensibilidades térmica, dolorosa e tátil ${ }^{10}$.

A ausência desses materiais dificulta os processos de diagnóstico clínico realizado pelo médico e o monitoramento realizado pela equipe. No caso da enfermagem, a enfermeira avalia e registra o grau de incapacidade física em prontuários e formulários, no diagnóstico e no acompanhamento dos pacientes com hanseníase, como também realiza o exame dermatoneurológico em todos os contatos intradomiciliares dos casos novos.

A conquista da confiança do paciente com hanseníase é papel de todos da equipe, que devem se empenhar em fortalecer o vínculo. O enfermeiro, em especial, usa o momento da consulta de enfermagem para realizar essa aproximação sabiamente. A utilização de técnicas pelos entrevistados vem aprimorando esse contato por meio de estratégias especiais, de conhecimentos particularizados, de disponibilidade de tempo e de alguns materiais específicos. A OMS, quando lançou a Estratégia Global 20162020, teve como um dos focos as recomendações operacionais estratégicas visando reduzir as incapacidades físicas desenvolvidas pela detecção tardia da hanseníase ${ }^{1}$.

Quando os enfermeiros foram indagados quanto à realização da prevenção de incapacidades, as respostas referiram-se à ausência de materiais específicos para o exame dermatoneurológico. Ainda, foi possível identificar, segundo os relatos, o quão imprescindível é a disponibilidade de um local silencioso e com equipamentos específicos ao atendimento do paciente com hanseníase.

[...] um dos desafios encontrados é a realização dos testes de sensibilidade, pois nas unidades não temos o estesiômetro, o qual é um dos recursos materiais que não está disponível [...] (E2)

[...] os desafios enfrentados são relacionados aos instrumentos pra fazer o exame físico e os monofilamentos que a unidade básica não possui, além dos tubos de ensaio para fazer os testes de sensibilidade térmica, ou qualquer outro material que possa ser utilizado para essa finalidade $[\ldots](E 4)$

Foi possível perceber que a falta de material impossibilita a realização completa e precisa de todos os itens dos testes de sensibilidade. A referida avaliação possui um papel importante logo após o diagnóstico médico e durante todo o período de https://doi.org/10.31011/reaid-2021-v.95-n.36-art.1157 Rev Enferm Atual In Derme v. 95, n. 36, 2021 e-021151 
tratamento atentando para um acompanhamento efetivo de prevenção das lesões dos nervos periféricos ${ }^{10}$. A detecção precoce dos distúrbios da função nervosa possibilita uma oportuna intervenção da equipe médica a fim de evitar uma perda progressiva e permanente da funcionalidade do nervo afetado. O poder incapacitante da hanseníase tem desdobramentos de natureza econômica, social e psicológica. Saliente-se que a doença incapacitante interfere na capacidade produtiva, na qualidade de vida e na vida social do indivíduo, contribuindo para traumas psicológicos e para perdas econômicas.

O exame realizado para testar a sensibilidade cutânea com os monofilamentos de Semmes-Weinstein tem-se apresentado de fácil execução e confiável quanto aos resultados. A utilização clínica rotineira do estesiômetro confere maior precisão ao teste, ao monitoramento da evolução da lesão nervosa periférica e à quantificação do grau de perda sensitiva em consequência da hanseníase ${ }^{5}$.

No tocante a doenças transmissíveis, a hanseníase configura-se uma das maiores causadoras de neuropatia periférica e incapacidades em todo mundo ${ }^{10}$. $\mathrm{E}$ vale destacar que esta é uma das principais causas de incapacidades que podiam ser evitadas a nível mundial ${ }^{11}$.

Neste contexto, a realização do exame dermatoneurológico desempenha de maneira simples uma avaliação neurológica atual e padronizada dos testes de sensibilidade, da força muscular e da palpação dos nervos periféricos, permitindo acompanhamento monitorizado, registro e intercâmbio de $\operatorname{dados}^{12}$. A disponibilização de formulário para roteiro e periocidade pré-determinada como no ato diagnóstico, na apresentação de queixas, no decorrer do tratamento em intervalos de no máximo um ano e na alta por cura $^{13}$.

\section{Intercorrências no atendimento ao paciente com hanseníase}

A hanseníase tem cura e dispõe de tratamento gratuito pelo SUS. O tratamento compreende terapia específica para eliminar o bacilo do organismo do paciente acometido, evitando complicações imunológicas e deformidades físicas incapacitantes. A equipe de saúde responsável atua desenvolvendo ações de promoção da saúde, prevenção de agravos e reabilitação psicossocial. Além disso, deve ser realizada a notificação compulsória à autoridade de saúde responsável e toda evolução do tratamento informada ${ }^{14}$.

A jornada terapêutica da hanseníase tem um longo período, podendo se estender por vários meses. Durante esse período de tratamento, podem surgir intercorrências, advindas da medicação, do estado imunológico do paciente e da própria doença 
em si. O processo de adoecer para o portador de hanseníase abrange aspectos complexos como a falta de conhecimento ou ideias antigas e preconceituosas relacionadas a questões sociais, culturais, biológicas e emocionais ${ }^{15}$.

Outro agravante da doença é representado pela ocorrência de surtos reacionais ao longo do tratamento, nos quais episódios inflamatórios se intercalam no curso crônico da hanseníase, podendo ser mais agressivos ou não. Os indivíduos ficam sujeitos à ocorrência de quadros reacionais antes, durante ou após o tratamento da doença, atingindo aproximadamente 25 a $30 \%$ dos indivíduos ${ }^{16}$. As interferências relatadas mais adiante pelos participantes foram variadas, sendo importante observar a ocorrência corriqueira delas na rotina de quem trabalha com esse tipo de paciente.

[...] outras pessoas têm reações hansênicas tipo I e II, concomitante com o aparecimento de novas lesões, diminuição da força muscular, alterações de sensibilidade das pernas essas são intercorrências que interferem num bom prognóstico da doença (E1).

O que ocorre mais são queixas relacionadas aos efeitos colaterais devido à medicação e com pouca frequência eventos adversos. Ah! Teve um paciente que desenvolveu quadro com fenômeno de Lúcio segundo o relatório médico (E6).

[...] na maioria das vezes o que a gente vê é reação tipo I. Então, o paciente recebe o tratamento e a gente consegue controlar esse paciente [...] (E3).
Quando o tratamento dos episódios reacionais é iniciado precocemente e realizado de forma adequada, reduz-se o dano neural em até $60 \%$, sendo imprescindível, portanto, que os profissionais de saúde tenham experiência e subsídios que facilitem a identificação, o diagnóstico e o tratamento desses quadros reacionais, prevenindo a ocorrência de incapacidades físicas ${ }^{16}$. Ainda, o relato muito enriquecedor citando um estado reacional raro conhecido como fenômeno de Lúcio mostra como é imprescindível essa discussão. Em 2021, um estudo de caso publicado no The New England Journal of Medicine apontou que o desenvolvimento de lesões cutâneas necrosantes graves em pacientes com hanseníase caracterizava-se como fenômeno de Lúcio, que é um quadro clínico incomum $^{17}$.

[...] às vezes o paciente faz uso de álcool, e por o tratamento ser prolongado ele não tem muita adesão ao tratamento, assim o profissional tem que ficar estimulando direto, sempre em interação com a equipe para criar vínculo e conseguir o retorno frequente [...] questão de intercorrência só quando ele não for à unidade, registrando a falta; na questão dos faltosos a agente de saúde vai à casa do paciente saber por que ele não compareceu a unidade (E5).

A busca dos faltosos bem como dos contatos é outra dificuldade, pois muitas vezes eles não comparecem [...] falta do transporte para buscar os pacientes e a supervisão da dose dos pacientes acamados (E2). 
Todos os pacientes com hanseníase passaram pela fase de comunicantes, portanto, são considerados de extrema importância epidemiológica em termos de endemia e passam a se tornar um grupo de risco suscetível do ponto de vista da cadeia do processo infeccioso ${ }^{18}$. Ademais, o acompanhamento deve ser realizado ambulatoriamente na rede de serviço público, onde o doente deve comparecer para consulta médica e de enfermagem, receber a dose supervisionada do tratamento PQT e realizar a avaliação de Prevenção de Incapacidades ${ }^{18}$.

É importante reconhecer que as neuropatias constituem a base de desenvolvimento de deficiências. Daí o diagnóstico precoce e o tratamento imediato serem muito importantes para impedir o processo de lesão nervosa, além de ser a intervenção mais essencial na prevenção e na limitação das deficiências. No entanto, deformidades e deficiências ocorrem na fase crônica da doença, devido à fraqueza e ao desgaste dos músculos inervados pelos nervos afetados, como por exemplo, mão em garra devido ao nervo ulnar ou pé caído devido à lesão do nervo fibular; outras ocorrências são queimaduras despercebidas, bolhas ou úlceras tróficas; no caso das úlceras tróficas, podem ocorrer na planta do pé, mãos e dedos devido à perda da sensação de dor; absorção de dedos e dedos dos pés vistos nos estágios finais da doença $^{19}$.
Outra questão relevante é a vulnerabilidade em que os pacientes e seus contatos estão inseridos na grande realidade social do Brasil. Em estudo realizado em Rondonópolis-MT, Brasil, foi constatado que a maioria dos contatos possui baixa renda e, apesar de boas condições de saneamento nas residências, grande parte possui fossa rudimentar, evidenciando um índice elevado de vulnerabilidade social ${ }^{20}$.

\section{Detecção precoce versus educação permanente}

A hanseníase é conhecida por ser uma doença que tem seu diagnóstico baseado na clínica. Contudo, o diagnóstico clínico ou até a identificação de sinais e sintomas resulta da experiência teórica e prática oriunda da vivência do profissional com os pacientes, com ênfase em cursos de educação continuada e práticas desenvolvidas em treinamentos e congressos.

Os profissionais enfermeiros sentem, muitas vezes, a necessidade de um centro de referência onde o paciente poderia dispor do serviço de média e alta complexidade fundamental ao longo do tratamento.

Apesar de o pessoal dizer que hanseníase é uma doença fácil de ser diagnosticada e fácil de ser tratada, não concordo com isso! É muito complexo, às vezes, ao realizar o exame físico no paciente nos deparamos com uma mancha com características da doença, porém acaba não se confirmando, realmente, difícil de diagnosticar porque 
têm que levar em consideração o nível cultural do paciente, às vezes, eles vão tão ansiosos achando que é hanseníase que eles mesmos sentindo dizem que não sentem (E5).

Nós não temos centro de referência para referenciar os portadores de hanseníase que têm uma gravidade maior e precisa de um acompanhamento mais específico (E1).

[...] e também a referência de pacientes na ocorrência de reação hansênica, pois não dispomos de um centro de referência $(E 2)$.

Os centros de referência possuem uma imagem de fundamental e potencial geradores de novos conhecimentos a fim de melhorar as condições de vida dos pacientes, pois desenvolvem ações de assistência, pesquisa e extensão ${ }^{21}$. No Ceará, existe um centro de referência nacional, localizado em Fortaleza, porém a distância para a região endêmica investigada impede que casos mais complexos sejam referenciados.

Essa realidade corresponde a uma orientação nacional das últimas duas décadas, pois o País tem realizado grandes esforços para introduzir a avaliação e a prevenção de incapacidades físicas, juntamente com a descentralização das atividades de controle da hanseníase na APS, almejando sempre o diagnóstico precoce ${ }^{22}$.

Ainda, o Programa Nacional de Controle da Hanseníase vem modificando seu perfil a partir da adesão a novas medidas tecnológicas em saúde e incorporação de diretrizes difundidas mundialmente ${ }^{21}$.
Embora, teoricamente, as ações de controle da hanseníase sejam implementadas em toda a rede de serviços públicos de saúde, a detecção passiva dos casos ainda é relatada como dificuldade evidenciada ${ }^{23}$.

A eliminação e o controle da hanseníase no País, enquanto questão imprescindível de saúde pública, direcionam todas as ações estratégicas para o aumento da detecção precoce e para alta por cura dos casos diagnosticados 5 .

Outro fator importante é a educação permanente, recentemente, tida como método mais utilizado pelos gestores para manter os profissionais atualizados e treinados sobre os temas inerentes a sua área de atuação com foco na resolução de problemas de saúde. A citada educação permanente é utilizada para processos de aprendizagem no trabalho com foco no êxito do programa como quesito técnico institucionalizado, valendo-se como ferramenta básica para o bom desempenho de qualquer profissional da assistência ${ }^{23}$. Os relatos que se seguem evidenciam ausência dessa rotina:

\footnotetext{
A hanseníase é um tema que no momento com relação ao município atual eu não fiz nenhum curso ainda, mas como eu já trabalhei em outras cidades e também no tempo da academia fiz diversos cursos de abordagem ao portador de hanseníase e tratamento, porém ofertado pelo município não fiz nenhum. Fui convidado pela coordenação do município para participar de um curso que irá acontecer em breve e já confirmei minha presença (E1).
} 
Em relação à parte prática e científica eu também acho relevante a questão de treinamento, educação continuada para está sempre assim renovando os conhecimentos aprendendo técnicas porque assim às vezes tem curso de capacitação, mas vai uma ou outra pessoa nunca vai todo mundo então uns acabam se beneficiando e outros não; aí com relação à hanseníase eu nunca fui nenhum tipo de treinamento. Aí tenho dificuldade nesse sentido, só que assim, o bom daqui é que a gente, o fisioterapeuta que a gente pode referenciar essa parte de reações de exame físico, ele faz. Aí diminui nesse sentido (E4).

O desempenho de papéis como a capacidade de julgamento clínico e a tomada de decisão do enfermeiro dependem do seu conhecimento teórico, experiência e aprimoramento continuado ${ }^{24}$. Assim, as equipes de ESF devem estar capacitadas de acordo com as necessidades do serviço e inseridas no contexto dos princípios do Sistema Único de Saúde ${ }^{25}$.

Um estudo apontou que o protagonismo assumido pelo profissional enfermeiro no envolvimento das ações de prevenção e controle realizadas pela equipe de saúde na ESF proporcionou um aumento nos casos diagnosticados e tratados ${ }^{26}$.

Vale destacar que, para os profissionais entrevistados, é de fundamental importância o fortalecimento da educação permanente, sendo de grande utilidade para realizar uma assistência qualidade ao paciente. No entanto, o programa funciona com a liberação de poucos profissionais para participar dos treinamentos gerando um https://doi.org/10.31011/reaid-2021-v.95-n.36-art.1157 Rev Enferm Atual In Derme v. 95, n. 36, 2021 e-021151 sentimento de insatisfação pelos não contemplados. Essa realidade mudou de forma parcial com a oferta de cursos online, especialmente pelo governo estadual, como forma de suprir a carência identificada. Porém, a ausência de módulos práticos e a discussão de realidades próprias de cada município podem ser prejudiciais para a capacitação continuada desses profissionais.

\section{CONSIDERAÇÕES FINAIS}

Diante dos resultados obtidos pelo presente estudo, foi possível conhecer as dificuldades enfrentadas pelos enfermeiros da ESF no tocante ao manejo dos pacientes com hanseníase. Dentre elas, vale destacar a falta de materiais específicos para realização dos testes dermatoneurológicos que dão suporte à detecção precoce, sendo assim, é válido apontar uma deficiência da gestão na inobservância à prioridade no controle da hanseníase em não fornecer instrumentos básicos para um atendimento adequado e eficiente.

Ainda, o preconceito e o estigma da hanseníase se fazem presentes nos dias atuais e precisa de investimento em tempo e em tecnologias educativas voltadas para a população. Torna-se um agravante a falta de conhecimento da população sobre a hanseníase o que vem a contribuir para reforçar essas duas vertentes. 
A detecção precoce é aceita como uma forma de quebrar a cadeia de transmissão mais rápida e prevenir incapacidades físicas. No entanto, apesar da hanseníase ser uma doença de diagnóstico médico com base na clínica apresentada pelo paciente, a sua captação pelos sinais e sintomas exige uma experiência prática desenvolvida com a doença pelos profissionais para atingirem um nível de segurança para uma abordagem eficaz.

A educação permanente é uma ferramenta auxiliar, sempre presente, utilizada para atualizar e aprimorar os conhecimentos dos profissionais da ESF, sendo também uma alternativa para suprir as lacunas no que se refere ao conhecimento dos profissionais sobre a hanseníase.

Nesse contexto, o estudo contribuiu ao apontar as dificuldades que impedem o aprimoramento do conhecimento sobre o manejo da hanseníase por enfermeiros que trabalham na ESF. Pode ser citada, como limitação do estudo, a amostra selecionada em um único município endêmico, o que impede a generalização dos achados. Além disso, evidencia-se a necessidade de mais estudos com profissionais de diferentes regiões do país com quadro endêmico para reforçar os discursos dos enfermeiros no que tange ao enfrentamento das dificuldades bem como o fortalecimento das ações de controle da hanseníase.

\section{REFERÊNCIAS}

1.World Health Organization (WHO). Global Leprosy Strategy 2016-2020: Accelerating towards a leprosy-free world; [Internet]. 2016 [acesso em 05 jan 2021]. Disponível em: http://apps.searo.who.int/PDS_DOCS/B5233. pdf

2.Basso MEM, Andrade RF, Ferrreira SRL. Tendência dos indicadores epidemiológicos da hanseníase em um estado endêmico da região amazônica. Rev Gaúcha Enferm.[Internet] 2021[acesso em 19 jun 2021];42:e20190520. Disponível em: https://seer.ufrgs.br/RevistaGauchadeEnferma gem/article/view/111387/60624

3.Secretaria da Saúde do Estado do Ceará. Situação epidemiológica da hanseníase. Informe Epidemiológico Hanseníase. [Internet]. 2021[acesso em 19 mai 2021]. Disponível em: https://www.saude.ce.gov.br/wpcontent/uploads/sites/9/2018/06/boletim_epid emiologico_hanseniase_20211901_v2.pdf

4.Pereira TM, Silva LMS, Dias MSA, Monteiro LD, Silva MRF, Alencar OM. Tendência temporal da hanseníase em região de alta endemicidade do Nordeste brasileiro. Rev Bras Enferm [Internet]. 2019 [acesso em 17 abr 2021] 72(5):1424-30. Disponível em: https://www.scielo.br/j/reben/a/RFcgSg3mFS $\mathrm{mKvDk}$ 7sq9TqLt/?format=pdf\&lang=pt.

5.Ministério da Saúde (BR). Guia prático sobre a hanseníase [internet]. 1. ed. Brasília (DF): Ministério da Saúde; 2017[acesso em 16 jun 2021]. Disponível em: https://portalarquivos2.saude.gov.br/images/p df/2017/novembro/22/Guia-Pratico-deHanseniase-WEB.pdf

6. Ministério da Saúde (BR), Secretaria de Vigilância em Saúde, Departamento de Vigilância das Doenças Transmissíveis. Diretrizes para vigilância, atenção e 
eliminação da hanseníase como problema de saúde pública, manual técnico-operacional [Internet]. Brasília: Ministério da Saúde; 2016 [acesso em 10 jun 2021]. Disponível em: http://portalarquivos.saude.gov.br/images/pdf/ 2016/fevereiro/04/diretrizes-eliminacaohanseniase 4fev16-web.pdf

7.Sousa GS, Silva RLF, Xavier MB. Hanseníase e Atenção Primária à Saúde: uma avaliação de estrutura do programa. Saúde em debate [Internet]. 2017[acesso em 15 jun 2021]; 41: 230-242. Disponível em: https://www.scielo.br/j/sdeb/a/GbTRqtP9Fmy TqxCSmVkLrZG/?lang=pt\&format=pdf.

8.Costa AKAN, Pfrimer IAH, Menezes AMF, Nascimento LB, Carmo Filho JR. Aspectos clínicos e epidemiológicos da hanseníase. Rev enferm UFPE on line. 2019 [acesso em 29 mai 2021]; 13(1):353-62. Disponível em: https://periodicos.ufpe.br/revistas/revistaenfer magem/article/view/236224.

9.Bardin L. Análise de Conteúdo. $1^{\mathrm{a}}$ ed. Lisboa: Almedina; 2011 [acesso em 28 out 2020]. 280 p.

10.Ministério da Saúde (BR). Secretaria de Atenção à Saúde. Departamento de Atenção Básica. Cadernos de Atenção Básica, no 21, Vigilância em Saúde: Dengue, Esquistossomose, Hanseníase, Malária, Tracoma e Tuberculose[Internet]. 2. ed. Revisada. Brasília (DF): Ministério da Saúde; 2008[acesso em 04 abr 2021]. Disponível em: http://vigilancia.saude.mg.gov.br/index.php/d ownload/cadernos-de-atencao-basica-no-21vigilancia-em-saude-dengue-

esquistossomose-hanseniase-malaria-tracomae-tuberculose/

11.Faria L, Calábria LK. Aspectos históricos e epidemiológicos da hanseníase em Minas Gerais. Rev Med Saude Brasilia [Internet]. 2018 [acesso em 23 abr 2021]; 06(3). Disponível em: https://portalrevistas.ucb.br/index.php/rmsbr/a rticle/view/8394.

12.Souza CDF de, Fernandes TRM de O, Matos TS, Ribeiro Filho JM, Almeida GKA de, Lima JCB, et al. Grau de incapacidade física na população idosa afetada pela hanseníase no estado da Bahia, Brasil. Acta Fisiátr. [Internet]. 2017 [acesso em 19 jun 2021]; 24(1). Disponível em: http://dx.doi.org/10.5935/01047795.20170006.

13.Duarte MTC, Ayres JA, Simonetti JP. Consulta de enfermagem ao portador de Hanseníase: proposta de um instrumento para aplicação do processo de enfermagem. Rev Bras Enferm [Internet]. 2008[acesso em 27 abr 2021]; 61(esp): 767-73. Disponível em: https://www.redalyc.org/pdf/2670/267019602 019.pdf.

14.Santos AR, Ignotti E. Prevenção de incapacidades física por hanseníase no Brasil: análise histórica. Ciência \& Saúde Coletiva [Internet]. 2020 [acesso em 6 jun 2021]; 25(10): 3731-3744. Disponível em: http://www.cienciaesaudecoletiva.com.br/arti gos/prevencao-de-incapacidade-fisica-porhanseniase-no-brasil-analisehistorica/17077?id=17077\&id=17077.

15.Silva Júnior GB, Daher EF, Pires Neto RJ, Pereira EDB, Meneses GC, Araújo SMHA, et al. Leprosy nephropathy: a review of clinical and histopathological features. Rev. Inst. Med. Trop. São Paulo [Internet]. 2015 [acesso em 10 jun 2021]; 57(1): 15-20. Disponível em:

http://www.scielo.br/pdf/rimtsp/v57n1/00364665-rimtsp-57-01-15.pdf.

16.Sangi KCC, Miranda LF, Spindola T, Leão AMM. Hanseníase e Estado Reacional: História de vida de pessoas acometidas. Rev enferm UERJ [Internet]. 2009[acesso em 19 mai 2021]; 17: 209-14. Disponível em: https://pesquisa.bvsalud.org/portal/resource/pt /lil-528341

17. Tajalli M, Wambier CG. Lucio's phenomenon. $\mathrm{N}$ Engl $\mathrm{J}$ Med [Internet]. 2021[acesso em 08 jun 2021]; 384(17): 1646. Disponível em: doi: 10.1056/NEJMicm2025081. PMID: 33913641. 
18. Vieira CSCA, Soares MT, Ribeiro CTSX, Silva LFG. Avaliação e controle de contatos faltosos de doentes com Hanseníase. Rev Bras Enferm [Internet]. 2008 [acesso em $16 \mathrm{fev}$ 2021];61(esp):682-8. Disponível em: http://www.scielo.br/scielo.php?pid=S003471 672008000700005\&script=sci_arttext.

19.Khadilkar SV, Patil SB, Shetty VP. Neuropathies of leprosy. J. Neurol. Sci. [Internet]. 2021 [acesso em 10 jun 2021];420:1-9. Disponível em: https://pubmed.ncbi.nlm.nih.gov/33360424/.

20. Santos DA da, Santos S, Ribeiro N, Goulart L, Mattos M, Ribeiro L, Olinda R. Vigilância de Contatos Domiciliares de Usuários com Hanseníase Menores de Quinze Anos em Município Hiperendêmico. REAID [Internet]. 2021 [acesso em 23 jun 2021];95(34):e-21027. Disponível em: https://revistaenfermagematual.com/index.ph p/revista/article/view/831

21. Oliveira MLW. Desafios para a efetividade das ações de controle da hanseníase. Cad Saúde Coletiva [Internet]. 2012 [acesso em 22 jun 2021];16(2):141-5. Disponível em: http://www.nesc.ufrj.br/cadernos/images/csc/ 2008_2/artigos/CSC_IESC_2008-

2_editorial.pdf

22. Ferreira IN. A hanseníase no contexto das doenças negligenciadas. In: Alves ED, Ferreira IN, Ferreira TL, organizadores. Hanseníase avanços e desafios [Internet]. Brasília: NESPROM; 2014 [acesso em 17 mai 2021]. p. 41-3. Disponível em: http://www.morhan.org.br/views/upload/hans eniaseavancoes.pdf

23.Brito KKG, Santana EMF, Andrade SSC, Peixoto VB, Nogueira JA, Soares MJGO. Análise epidemiológica da hanseníase em um estado endêmico do nordeste brasileiro. Rev Gaúcha Enferm. [Internet]. 2015 [acesso em 04 jun 2021]; 36: 24-30. Disponível em: https://www.scielo.br/j/rgenf/a/8qTp7t5k5Zw shmmrXfzPYHb/?format=pdf\&lang $=$ pt

24.Ministério da Saúde (BR). Secretaria de Vigilância em Saúde. Boletim Epidemiológico Hanseníase, verminoses e tracoma têm cura: a experiência de uma campanha integrada [Internet]. 2016 [acessado em 12 jan 2021]; 47(21): 1-10. Disponível em: http://portalms.saude.gov.br/boletinsepidemiologicos

25. Oliveira KS de, Arcoverde MAM, Deschutter JH, Silva AJ da, Zilly A, Silva Sobrinho RA da. Hanseníase em países fronteiriços na América do Sul: um estudo ecológico. Cogitare enferm. [Internet]. 2019 [acesso em 17 jun 2021]; 24. Disponível em: http://dx.doi.org/10.5380/ce.v24i0.64917.

26.Pinheiro JJG, Gomes SCS, Aquino DMC, Caldas AJM. Aptidões cognitivas e atitudinais do enfermeiro da atenção básica no controle da hanseníase. Rev. baiana enferm. [Internet]. 2017 [acesso em 27 mai 2021]; 31(2):e17257. Disponível em: https://periodicos.ufba.br/index.php/enfermag em/article/view/17257/14517.

\section{Autor correspondente}

Ana Alinne Gomes da Penha. Endereço: Rua Antônio Fernandes Lima, Centro, Farias Brito, Ceará, 63185-000. Telefone: (88) 35441131. Email: anaalinne.nurse@gmail.com

Submissão: 2021-06-24

Aprovado: 2021-10-04 